\title{
Waldenström Macroglobulinemia
}

\author{
Irene M. Ghobrial, MD and Thomas E. Witzig, MD* \\ Division of Hematology, Mayo Clinic, 200 First Street, Rochester, MN 55905, USA
}

\section{Opinion statement}

Waldenström macroglobulinemia (WM) is a low-grade lymphoproliferative disorder characterized by the presence of an immunoglobulin $\mathrm{M}$ monoclonal protein in the blood and monoclonal small lymphocytes and lymphoplasmacytoid cells in the marrow. The disease is uncommon and there is a lack of clear diagnostic criteria. WM is treatable but not curable and long-term survival is possible. Therefore, the treating physician needs to carefully balance the risks and benefits of treatment. Treatments are aimed at relieving symptoms resulting from marrow infiltration and the hyperviscosity syndrome. Therapies available for initiation of treatment include alkylating agents, purine nucleoside analogs, and rituximab. Chlorambucil has been the mainstay of treatment for many years and remains useful, especially in older patients. Rituximab has become an important new therapy for this disease because of its positive treatment responses, acceptable toxicity, and lack of therapy-associated myelosuppression and myelodysplasia. Currently, rituximab is being combined with chemotherapy. Other options of treatment include interferon and corticosteroids. Emerging therapies include stem cell transplantation (autologous and allogeneic) for younger patients. Currently, there are few comparative data on which to state an absolute opinion concerning the best available treatment for patients with WM.

\section{Introduction}

Waldenström macroglobulinemia (WM) was first described by Jan Waldenström in 1944 [1] and is classified in the Revised European-American Classification/World Health Organization classification as a lymphoplasmacytic lymphoma [2]. It is believed that these cells originate from post germinal center B cells after somatic hypermutation and before class switching [3]. It is characterized by a lymphoplasmacytic infiltrate in the bone marrow and an immunoglobulin (Ig) M protein in the blood.

The diagnosis of WM is based on the presence of classic signs and symptoms of the disease, monoclonal lymphoplasmacytic infiltrate in the bone marrow, and IgM monoclonal protein in the blood.

Classic signs and symptoms of the disease include fatigue and bleeding, usually in the form of chronic epistaxis and gingival oozing. The fatigue is usually caused by anemia and the bleeding is caused by hyperviscosity. Lymphadenopathy and splenomegaly are observed in approximately $25 \%$ of cases and are not nearly as prominent as found in non-Hodgkin lymphoma. Unusual manifestations include peripheral neuropathy and the development of primary amyloidosis $\left[4,5^{\bullet}\right]$.

The typical bone marrow infiltrate consists of lymphoplasmacytoid cells with a small percentage of plasma cells; however, the morphology can range from predominantly 
lymphocytic to lymphoplasmacytoid to overt plasma cells [6]. The malignant cells express the pan B-cell surface markers (CD19, CD20, CD22) in association with monoclonal light chain expression on the cell surface and cytoplasm. CD138 is expressed by the plasma cells present in the infiltrate. Most of the cases do not express the T cell marker CD5. These immunophenotyping markers can help differentiate WM from other lymphoproliferative diseases such as B-chronic lymphocytic leukemia (CLL), and mantle cell lymphoma (Fig. 1) [7]. Many chromosomal abnormalities have been described in WM, but none of the chromosomal abnormalities are specific. Therefore, it is not necessary to perform cytogenetic analysis, except in cases where the diagnosis is unclear.

The minimal concentration of IgM protein recommended as a diagnostic criterion is $1.5 \mathrm{gm} /$ $\mathrm{dL}$. However, the median level of IgM in symptomatic patients is usually higher. In a recent clinical trial by Kyle and Garton [8], the median level was $4.2 \mathrm{~g} / \mathrm{dL}$ in untreated symptomatic patients. Furthermore, $40 \%$ to $80 \%$ of patients with WM have monoclonal light chains (Bence Jones proteins) detected in the urine [8]. Figure 2 shows the differential diagnosis of an IgM monoclonal protein.

\section{DIFFERENTIATING WM FROM MONOCLONAL GAMMOPATHY OF UNDETERMINED SIGNIFICANCE AND SMOLDERING WM}

Immunoglobulin $\mathrm{M}$ monoclonal gammopathy is a spectrum of diseases that ranges from asymptomatic IgM monoclonal gammopathy of undetermined significance (MGUS) to smoldering WM to symptomatic WM requiring treatment. MGUS can be differentiated from WM by the following criteria (Fig. 3): the absence of anemia, hepatosplenomegaly, lymphadenopathy, systemic symptoms, minimal lymphocytic infiltration of the bone marrow; and serum IgM concentration less than $2.0 \mathrm{~g} / \mathrm{dL}$, which does not increase with time.

Kyle et al. [8,9••] have shown in a long-term follow-up of 242 patients with IgM MGUS that $9 \%$ of patients developed WM and 7\% developed lymphoma, CLL, or amyloidosis.

\section{DIFFERENTIATING WM FROM OTHER B-CELL LYMPHOPROLIFERATIVE DISORDERS}

Waldenström macroglobulinemia must be differentiated from other IgM related disorders such as B-CLL, non-Hodgkin's lymphoma, heavy chain disease, primary specific amyloidosis, and rarely multiple myeloma (MM). Occasionally, it may be difficult to differentiate other lymphoproliferative disorders such as marginal zone lymphoma from WM. These can usually be resolved with immunophenotyping, expert morphology interpretation, and integration of the pathologic and clinical features [7] (Fig. 1).

\section{HYPERVISCOSITY}

Hyperviscosity is the hallmark of WM, but it only occurs in approximately 15\% of the cases. It is characterized by oronasal bleeding, retinal hemorrhage, and neurologic abnormalities. Symptoms usually occur at serum viscosity values more than $4 \mathrm{cps}$, and almost all patients with viscosity greater than $8 \mathrm{cps}$ are symptomatic. The syndrome does not always correlate with the serum viscosity or a specific level of $\mathrm{M}$ protein and the decision to perform a plasmapheresis should be based not only on the lab values but also the symptoms of the patients and the signs detected on physical exam. Patients with WM and new onset mental confusion should undergo emergency plasmapheresis because this can produce remarkable clinical improvement [10]. 


\section{Treatment}

- As in MM, WM seems to evolve from a continuum with MGUS at one end of the spectrum and symptomatic WM at the opposite end. Because WM is an incurable disease, early treatment of asymptomatic patients is not warranted because the goal of therapy is the relief of symptoms and prevention of end organ damage. Asymptomatic patients can be closely observed until disease progression occurs clinically and by laboratory measures [8].

\section{Pharmacologic treatment}

- The options of treatment available for patients with WM are summarized in Table 1.

\section{Alkylating agents}

Chlorambucil: Oral alkylating agents have been the most commonly used agent for the last four decades. The response rate with chlorambucil is high at $79 \%$, the drug is well-tolerated, and the median overall survival time is 5.4 years in a recent large series [11•]. Responses may occur slowly and take up to 6 months to occur.

Standard dosage: $0.1 \mathrm{mg} / \mathrm{kg}$ per day orally before meals. Most patients require dose reductions and the typical daily dose is 4 to $6 \mathrm{mg} / \mathrm{d}$.

Contraindications: Hypersensitivity to chlorambucil or any component of the formulation; pregnancy.

Main drug interactions: Patients may experience impaired immune response to vaccines and possible infection after administration of live vaccines in patients receiving immunosuppressants.

Main side effects: Myelosuppression is the main side effect. Significant nausea and drug rashes is unusual. The most important long-term side effect is myelodysplasia or acute leukemia, which was observed in $9 \%$ of patients [11•].

\section{Other alkylating agents in combinations}

- The VBMCP (vincristine, carmustine, melphalan, cyclophosphamide, and prednisone) regimen has also been used in WM. In one study, 33 patients received VBMCP every 5 weeks for 2 years, followed by maintenance therapy every 10 weeks [12]. Twenty-one (64\%) partial responses and six minor responses were observed.

- Other agents have also been used, such as cyclophosphamide, doxorubicin, and prednisone.

\section{Combinations of alkylating agents}

- Laurencet et al. [13] administered cladribine (2-CDA) $0.1 \mathrm{mg} / \mathrm{kg}$ per day subcutaneous for three consecutive days with intravenous cyclophosphamide 500 $\mathrm{mg} / \mathrm{m}^{2}$ and oral prednisone monthly for up to six courses. The overall response rate (ORR) was $88 \%$. Severe myelosuppression with grade 4 neutropenia occurred in $11 \%$ of patients and prolonged thrombocytopenia resulted in failure to receive further cycles of chemotherapy in $31 \%$ of patients. 


\section{Purine nucleoside analogs}

Fludarabine: Because purine nucleoside analogs (PNA) have been useful in other lowgrade lymphoproliferative malignancies, it seemed rational to test them in WM. The largest study of fludarabine in WM enrolled 231 patients. One hundred sixty-seven patients were previously untreated, and 49 did not receive fludarabine because they were asymptomatic. [14•]. The ORR was 36\% (2\% complete remission) with no difference between patients who received previous therapies versus those who had no prior treatments.

Standard dosage: A dose between 25 to $30 \mathrm{mg} / \mathrm{m}^{2}$ daily for 5 days, repeated every 28 days. Patients receive four to eight cycles of fludarabine.

Contraindications: Hypersensitivity to fludarabine, severe infections, and severe renal impairment (creatinine clearance $<30 \mathrm{~mL} / \mathrm{min} / \mathrm{m} \mathrm{[2]).}$

Main drug interactions: Live vaccines and rotavirus vaccine should not be used in these patients.

Main side effects: The primary toxicity is myelosuppression and depression of T cells, resulting in a $4 \%$ mortality from infections. Other side effects include hemolytic anemia temporally related to fludarabine treatment and neurotoxicity.

Special points: Because of the known myelosuppressive and immunosuppressive effects of fludarabine, the number of doses/cycle or the number of cycles should be reduced if possible.

Cladribine: Several studies have used 2-CDA as first-line therapy or in previously treated patients. The largest study treated 29 patients with WM with a continuous intravenous infusion of $0.1 \mathrm{mg} / \mathrm{kg}$ per day 2-CDA for 7 days for a total of two courses [15]. They reported an ORR of 59\% (100\% in previously untreated patients and $40 \%$ for relapsed patients).

Standard dosage: $0.1 \mathrm{mg} / \mathrm{kg}$ intravenously every day for 7 days, repeated every month.

Contraindications: Patients with pre-existing myelosuppression or infection.

Main drug interactions: Drugs that can cause myelosuppression or concurrent radiation therapy can lead to severe bone marrow depression when used with 2-CDA. Dose adjustment is necessary when these agents are used with 2-CDA or at a later date. Killed virus vaccines should not be used because the patient's antibody response to the vaccine may be decreased because of the immunosuppression induced by the drug. Live vaccines are also contraindicated because the patient's immune response may be suppressed leading to increased replication of the virus, increased side effects, and decreased antibody response to the vaccine.

Main side effects: Myelosuppression is the main toxicity. Patients may also develop infections, bacte rial, protozoal, fungal, and viral (herpes zoster) secondary to cellular immunity suppression. 2-CDA may cause prolonged depression of CD4 and CD8 lymphocyte counts with delayed recovery of 6 to 12 months.

Special points: Based on the studies involving WM, 2-CDA should be limited to two cycles to avoid profound myelosuppression. 
Rituximab: Rituximab is a human chimeric unlabeled monoclonal antibody directed against CD20. Because most of the WM cells express CD20 and because it is generally well tolerated and not myelosuppressive, rituximab has become an important new agent in the treatment of WM. Several studies have shown ORRs between $44 \%$ to $60 \%$ with no difference between previously untreated or treated patients $[16,17,18 \bullet, 19]$. The median time to response in a recent study by Dimopoulos et al. [18•] was 3.3 months, and the median time to progression was 16 months. Furthermore, the drug is not associated with myelosuppression and myelodysplasia, such as the alkylating agents and PNAs.

Standard dosage: $375 \mathrm{mg} / \mathrm{m}^{2}$ intravenously once weekly for four doses.

Contraindications: Hypersensitivity to murine proteins.

Main drug interactions: Severe renal toxicity has occurred in patients administered the combination rituximab and cisplatin. As with the other chemotherapies, live vaccines should not be given concomitantly with rituximab.

Main side effects: Most of the side effects of rituximab occur during the infusion and more frequently during the first infusion. Myelosuppression is unusual.

Special points: Clinical responses may be delayed for several months after completion of therapy with rituximab. Patients with initial M protein increases after being treated with rituximab have been demonstrated to eventually respond with further observation [18•]. Anti-CD20 radioimmunoconjugates have shown superior ORR in relapsed low grade nonHodgkin's lymphoma compared to rituximab, and they need to be tested in patients with WM in the future [20].

\section{Corticosteroids}

- Steroids can be useful in the treatment of WM in patients with severe cytopenias that preclude the use of cytotoxic drugs or in patients with cryoglobulin associated vasculitis. Reports on limited numbers of patients have shown effectiveness in the reduction of the $\mathrm{M}$ protein and viscosity with steroids.

\section{Interferon}

- Interferon may have some clinical use in some patients with WM, although it is usually associated with side effects. The largest study using interferon was a phase II trial that included 88 patients with $43 \%$ of them classified as having WM. The treatment consisted of $3 \times 10^{6} \mathrm{~m} /$ day for 1 month and then three times a week as a maintenance therapy. The ORR was 32\% (> 50\% M-component reduction), and an additional $16 \%$ of patients had a $25 \%$ to $50 \%$ M-protein reduction. Patients have shown response to interferon with a decrease in the bone marrow infiltration, increased hemoglobin, and improvement in hyperviscosity symptoms.

\section{Radiation therapy}

- Unlike MM, WM is rarely diagnosed with lytic lesions. Therefore, radiation therapy is not usually required.

\section{Bone marrow biopsy}

- A unilateral bone marrow aspirate and biopsy is important in the diagnosis of WM and subsequently if the clinical status changes. It typically shows infiltration with monoclonal lymphoplasmacytic cells [21-23]. Mast cells may also be present in 
association with the malignant clone $[7,22,23]$. Flow cytometry will show these cells expressing the pan-B cell surface markers (CD19, CD20, CD22). CD138 is present on the plasma cell component. These cells also express monoclonal light chain on the cell surface and cytoplasm [7,24-27], and CD5, CD10, and CD23 are usually absent in WM [6]. This immunophenotype, with the typical morphology, can help differentiate WM from other lymphoproliferative disorders such as BCLL, mantle cell lymphoma, marginal zone, and follicular B-cell lymphoma.

\section{Plasma exchange}

- Patients with symptoms of hyperviscosity should undergo emergency plasmapheresis irrespective of the viscosity value or if the laboratory test is unavailable. However, hyperviscosity without symptoms is not an indication for plasmapheresis. Once a patient develops symptoms at a certain IgM protein level, that level may be used in the future for that patient for monitoring of hyperviscosity syndrome.

\section{Plasmapheresis}

Standard procedure: The total plasma volume to be exchanged in an adult is approximately 3 to $4 \mathrm{~L}$. It is preferred to use albumin rather than plasma in this condition. Plasma exchange is usually performed using centrifugation devices. Another possible way is the use of a highly permeable filter with standard hemodialysis equipment. A central line with a rigid dual lumen catheter is required (Quinton-Mahurkar catheter, Quinton Instrument Company, Seattle, WA). Plasmapheresis is repeated daily until symptoms subside and the viscosity is lowered. Because most of the IgM protein is intravascular, one or two procedures are usually sufficient to reduce the IgM level. Symptoms subside with the lowering of serum viscosity unless irreversible changes have occurred such as venous thrombosis of the retinal vein or other sites.

Main side effects: Side effects are more common with fresh frozen plasma than with albumin replacement (20\% vs $1.4 \%$ ). The most frequent problems are paresthesias, muscle cramps, and urticaria. Other complications include hypotension because of volume depletion, citrate induced hypocalcemia and metabolic alkalosis, and complications related to vascular access. Volume overload and dyspnea can occur because the volume of plasma calculated for one volume exchange is usually greater than the volume of plasma required for these patients, and hyperviscosity is usually associ ated with an increased plasma volume. More serious complications, such as severe anaphylactoid reactions, typically follow the administration of fresh frozen plasma and other plasma-containing replacement fluid. The overall incidence of death is $0.03 \%$ to $0.05 \%$.

Special points: Red blood cell transfusions can lead to an increase in viscosity and worsening of the condition; therefore, there is no need for over correction of anemia in these patients. If transfusions are required, then plasmapheresis should be initiated before transfusions in these patients.

\section{Surgery}

- Splenectomy is effective in patients who develop hypersplenic syndrome or who have dominant disease in the spleen [28]. Several case reports have demonstrated disappearance of monoclonal protein and prolonged disease-free survival after splenectomy [29]. Furthermore, one patient was reported to have Richter's transformation in the spleen and had disease control with splenectomy [30]. 


\section{Other treatments}

\section{Thalidomide}

- Thalidomide has emerged as an immunomodulatory and antiangiogenic novel therapy for patients with myeloma, thus it has been used in patients with WM. It can inhibit angiogenesis and induce apoptosis of established neovasculature in experimental models. It has shown significant activity in refractory myeloma, and in previously untreated myeloma. However, the first phase II trial reported by Dimopoulos et al. [31] (20 patients, with 10 treated previously) showed only a $25 \%$ ORR and a time to progression of less than 3 months. Therefore, thalidomide used as a single agent appears less promising than the results with MM.

\section{Stem cell transplantation}

- Very few reports have been published on transplantation in WM, thus it remains an experimental treatment in this disease.

- Autologous transplantation is limited because of the previous heavy exposure to alkylating agents and the extensive marrow involvement. Therefore, if stem cell transplantation is contemplated, physicians should harvest the patient early, before extensive exposure to alkylating agents or PNAs. Desikan et al. [32] reported on the use of single-agent melphalan in five patients $\left(200 \mathrm{mg} / \mathrm{m}^{2}\right)$ and melphalan $(140$ $\mathrm{mg} / \mathrm{m}^{2}$ ) with total body irradiation in one patient as conditioning regimens. A subsequent study of seven patients using high-dose cyclophosphamide and total body irradiation as a conditioning regimen showed a decrease in the serum protein in all patients and a time to progression of 3 to 30 months [33].

- Allogeneic transplantation is rare because of the older median age of the patients.

\section{Acknowledgments}

Support is, in part, by grant CA097274 and the Research Fund for Waldenström Macroglobulinemia.

\section{References and Recommended Reading}

Papers of particular interest, published recently, have been highlighted as:

- Of importance

•• Of major importance

1. Waldenström J. Incipient myelomatosis or "essential" hyperglobulinemia with fibrinogenopenia: a new syndrome? Acta Med Scand. 1944; 117:216-222.

2. Harris NL, Jaffe ES, Diebold J, et al. World Health Organization classification of neoplastic diseases of the hematopoietic and lymphoid tissues: report of the Clinical Advisory Committee meeting-Airlie House, Virginia, November 1997. J Clin Oncol. 1999; 17:3835-3849. [PubMed: 10577857]

3. Wagner S, Martinelli V, Luzzato L. Similar patterns of somatic hypermutation in hairy cell leukemia, prolymphocytic leukemia, Waldenström's macroglobulinemia and myeloma. Blood. 1994; 83:3647-3653. [PubMed: 8204889]

4. Dimopoulos MA, Panayiotidis P, Moulopoulos LA, et al. Waldenström's macroglobulinemia: clinical features, complications, and management. J Clin Oncol. 2000; 18:214-226. [PubMed: 10623712]

5•. Garcia-Sanz R, Montoto S, Torrequebrada A, et al. Waldenström macroglobulinaemia: presenting features and outcome in a series with 217 cases. Br J Haematol. 2001; 115:575-582. This is a report of 217 patients presenting with WM in Spain over 10 years. It presents the clinical 
features, the prognostic factors, the treatments received, and the causes of death of these patients. [PubMed: 11736938]

6. Andriko JA, Aguilera NS, Chu WS, et al. Waldenström's macroglobulinemia: a clinicopathologic study of 22 cases. Cancer. 1997; 80:1926-1935. [PubMed: 9366295]

7. Pangalis G, Angelopoulou M, Vassilakopoulos P. B-chronic lymphocytic leukemia, small lymphocytic lymphoma, and lymphoplasmacytic lymphoma, including Waldenström's macroglobulinemia: a clinical, morphologic, and biologic spectrum of similar disorders. Semin Hematol. 1999; 36:104-114. [PubMed: 10319379]

8. Kyle RA, Garton JP. The spectrum of IgM monoclonal gammopathy in 430 cases. Mayo Clin Proc. 1987; 62:719-731. [PubMed: 3110508]

9••. Kyle RA, Therneau TM, Rajkumar SV, et al. A long-term study of prognosis in monoclonal gammopathy of undetermined significance. N Engl J Med. 2002; 346:564-569. Monoclonal gammopathy of undetermined significance progressed in 115 of 1384 patients to MM, IgM lymphoma, primary amyloidosis, macroglobulinemia, chronic lymphocytic leukemia, or plasmacytoma. This is the largest follow-up study of patients with MGUS. [PubMed: 11856795]

10. Gertz MA, Kyle RA. Hyperviscosity syndrome. J Intensive Care. 1995; 10:128-141.

11 . Kyle RA, Greipp PR, Gertz MA, et al. Waldenström's macroglobulinaemia: a prospective study comparing daily with intermittent oral chlorambucil. Br J Haematol. 2000; 108:737-742. Seventy-nine percent of 24 patients administered continuous therapy had an objective improvement by reduction of serum M protein or increase in hemoglobin. Sixty-eight percent of 22 patients administered chlorambucil intermittently had an objective response. This is one of the most important studies showing effectiveness of chlorambucil in patients with WM. [PubMed: 10792277]

12. Case DC Jr, Ervin TJ, Boyd MA, et al. Waldenström's macroglobulinemia: long-term results with the M-2 protocol. Cancer Invest. 1991; 9:1-7. [PubMed: 1901509]

13. Laurencet FM, Zulian GB, Guetty-Alberto M, et al. Cladribine with cyclophosphamide and prednisone in the management of low-grade lymphoproliferative malignancies. Br J Cancer. 1999; 79:1215-1219. [PubMed: 10098762]

14•. Dhodapkar MV, Jacobson JL, Gertz MA, et al. Prognostic factors and response to fludarabine therapy in patients with Waldenström macroglobulinemia: results of United States intergroup trial (Southwest Oncology Group S9003). Blood. 2001; 98:41-48. One hundred eighty-two patients with symptomatic or progressive disease were treated with four to eight cycles of therapy with fludarabine. The overall rate of response to therapy was $36 \%$, with $2 \%$ complete remission. Patients who were 70 years or older had a substantially lower likelihood of response. [PubMed: $11418461]$

15. Dimopoulos MA, Kantarjian H, Estey E, et al. Treatment of Waldenström macroglobulinemia with 2-chlorode-oxyadenosine. Ann Intern Med. 1993; 118:195-198. [PubMed: 8093333]

16. Byrd JC, White CA, Link B, et al. Rituximab therapy in Waldenström's macroglobulinemia: preliminary evidence of clinical activity. Ann Oncol. 1999; 10:1525-1527. [PubMed: 10643548]

17. Treon SP, Agus DB, Link B, et al. CD20-directed antibody-mediated immunotherapy induces responses and facilitates hematologic recovery in patients with Waldenström's macroglobulinemia. J Immunother. 2001; 24:272-279.

18•. Dimopoulos MA, Zervas C, Zomas A, et al. Treatment of Waldenström's macroglobulinemia with rituximab. J Clin Oncol. 2002; 20:2327-2333. This is a prospective phase II study to define the activity of rituximab in patients with WM. Responses occurred in $40 \%$ of previously untreated patients and 50\% of pretreated patients. [PubMed: 11981004]

19. Treon SP, Anderson KC. The use of rituximab in the treatment of malignant and nonmalignant plasma cell disorders. Semin Oncol. 2000; 27(Suppl 12):79-85. [PubMed: 11226004]

20. Witzig TE, Gordon LI, Cabanillas F, et al. Randomized controlled trial of yttrium-90-labeled ibritumomab tiuxetan radioimmunotherapy versus rituximab immunotherapy for patients with relapsed or refractory low-grade, follicular, or transformed B-cell non-Hodgkin's lymphoma. J Clin Oncol. 2002; 20:2453-2463. [PubMed: 12011122]

21. Kapff, C.; Jandl, J. Blood: Atlas and Sourcebook of Hematology. Boston: Little Brown; 1991. 
22. Dutcher T, Fahey J. The histopathology of macroglobulinemia of Waldenström. J Natl Cancer Inst. 1959; 22:887-917. [PubMed: 13655069]

23. Bartl R, Frisch B, Mahl G, et al. Bone marrow histology in Waldenström's macroglobulinaemia. Clinical relevance of subtype recognition. Scand J Haematol. 1983; 31:359-375. [PubMed: 6414077]

24. Owen R, Johnson S, Morgan G. Waldenström's macroglobulinemia: laboratory diagnosis and treatment. Hematol Oncol. 2000; 18:41-49. [PubMed: 10960874]

25. Owen RG, Barrans SL, Richards SJ, et al. Waldenström macroglobulinemia. Development of diagnostic criteria and identification of prognostic factors. Am J Clin Pathol. 2001; 116:420-428. [PubMed: 11554171]

26. Kucharska-Pulczynska M, Ellegard J, Hokland P. Analysis of leukocyte differentiation antigens in blood and bone marrow from patients with Waldenström macroglobulinemia. Br J Haematol. 1987; 65:395-399. [PubMed: 3107610]

27. Feiner H, Rizk C, Finfer M. IgM monoclonal gammopathy/Waldenström's macroglobulinemia: a morphological and immunophenotypic study of the bone marrow. Mod Pathol. 1990; 3:348-356. [PubMed: 2114024]

28. Nagai M, Ikeda K, Nakamura H, et al. Splenectomy for a case with Waldenström macroglobulinemia with giant splenomegaly. Am J Hematol. 1991; 37:140. [PubMed: 1906242]

29. Humphrey JS, Conley CL. Durable complete remission of macroglobulinemia after splenectomy: a report of two cases and review of the literature. Am J Hematol. 1995; 48:262-266. [PubMed: 7717376]

30. Winter AJ, Obeid D, Jones EL. Long survival after splenic immunoblastic transformation of Waldenström's macroglobulinaemia. Br J Haematol. 1995; 91:412-414. [PubMed: 8547084]

31. Dimopoulos MA, Zomas A, Viniou NA, et al. Treatment of Waldenström's macroglobulinemia with thalidomide. J Clin Oncol. 2001; 19:3595-3601.

32. Desikan R, Dhodapkar M, Siegel D, et al. High-dose therapy with autologous haemopoietic stem cell support for Waldenström's macroglobulinaemia. Br J Haematol. 1999; 105:993-996. [PubMed: 10554812]

33. Dreger P, Glass B, Kuse R, et al. Myeloablative radio-chemotherapy followed by reinfusion of purged autologous stem cells for Waldenström's macroglobulinaemia. Br J Haematol. 1999; 106:115-118. [PubMed: 10444173] 


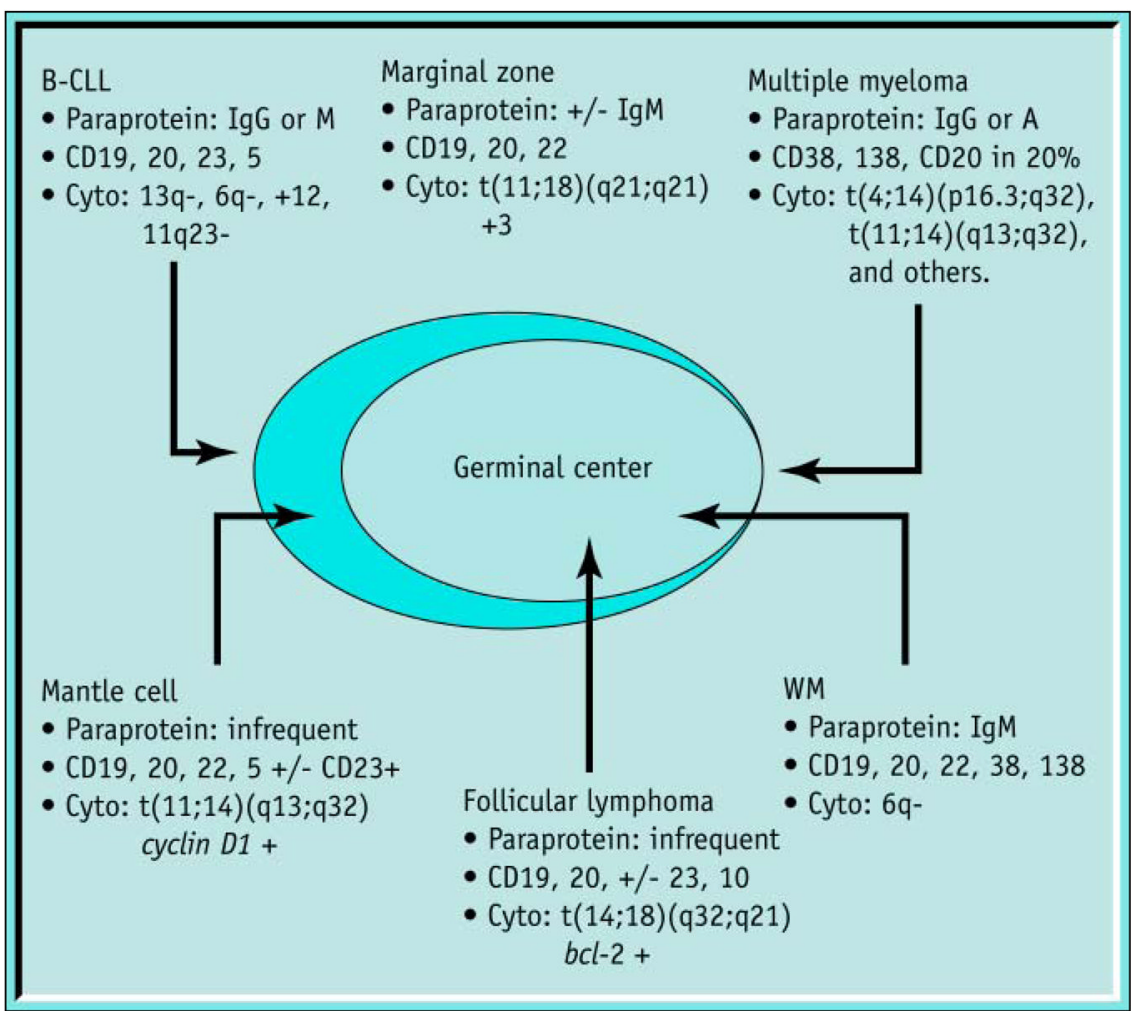

Figure 1.

The differential diagnosis of WM from other B-lymphoproliferative disorders, such as BCLL, mantle cell lymphoma, marginal zone lymphoma, follicular lymphoma, multiple myeloma, and amyloidosis. B-CLL-B-chronic lymphocytic leukemia; Cyto-cytochrome; Ig_immunoglobulin; WM-Waldenström macroglobulinemia. 


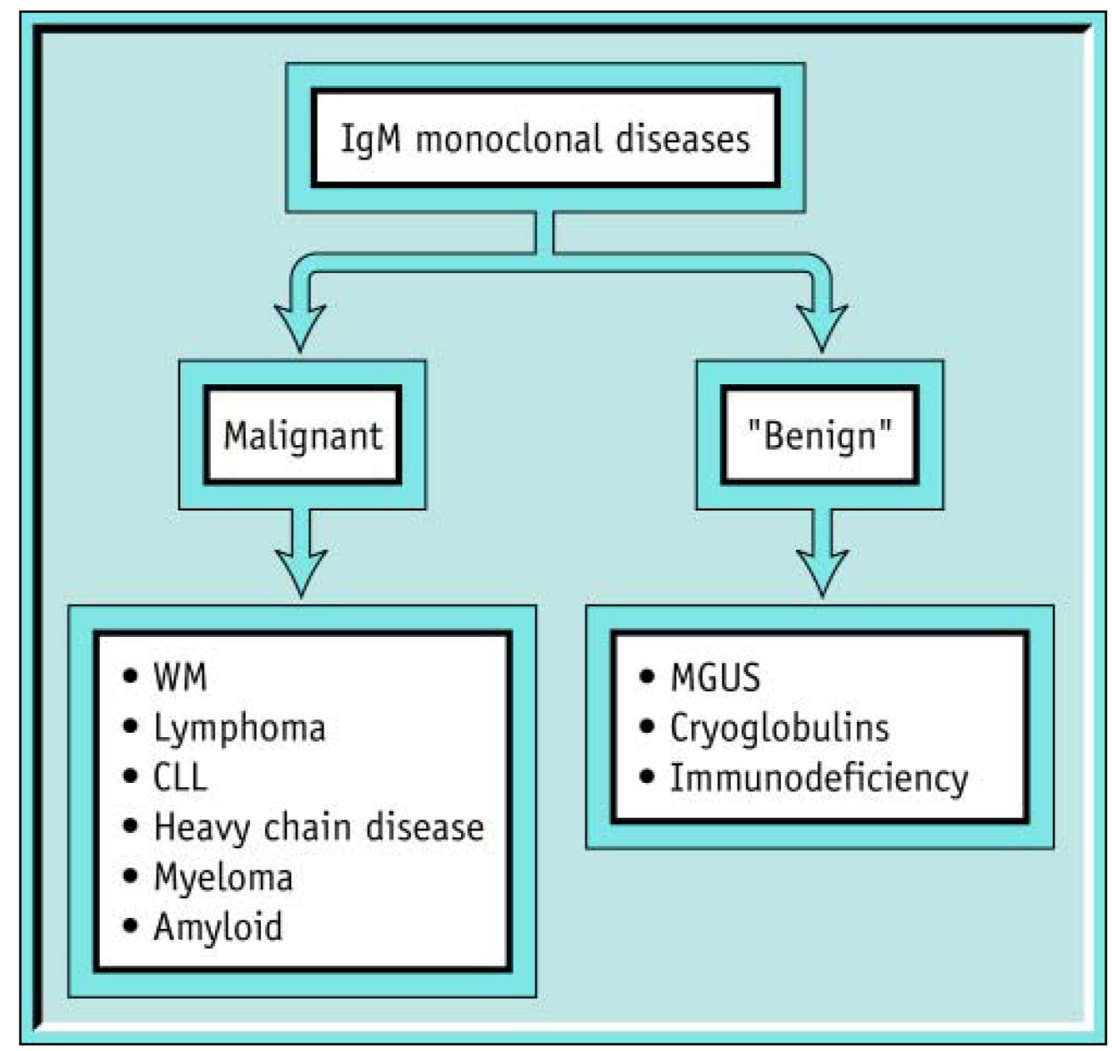

Figure 2.

The differential diagnosis of an IgM monoclonal protein includes malignant and benign causes. Malignant etiologies include WM, lymphoma, CLL, multiple myeloma, heavy chain disease, and amyloidosis. Benign etiologies include transient $\mathrm{M}$ proteins, monoclonal gammopathy of undetermined significance, cryoglobulins, and some immunodeficiency diseases. CLL_chronic lymphocytic leukemia; Ig_immunoglobulin; MGUS—monoclonal gammopathy of undetermined significance; WM-Waldenström macroglobulinemia. 


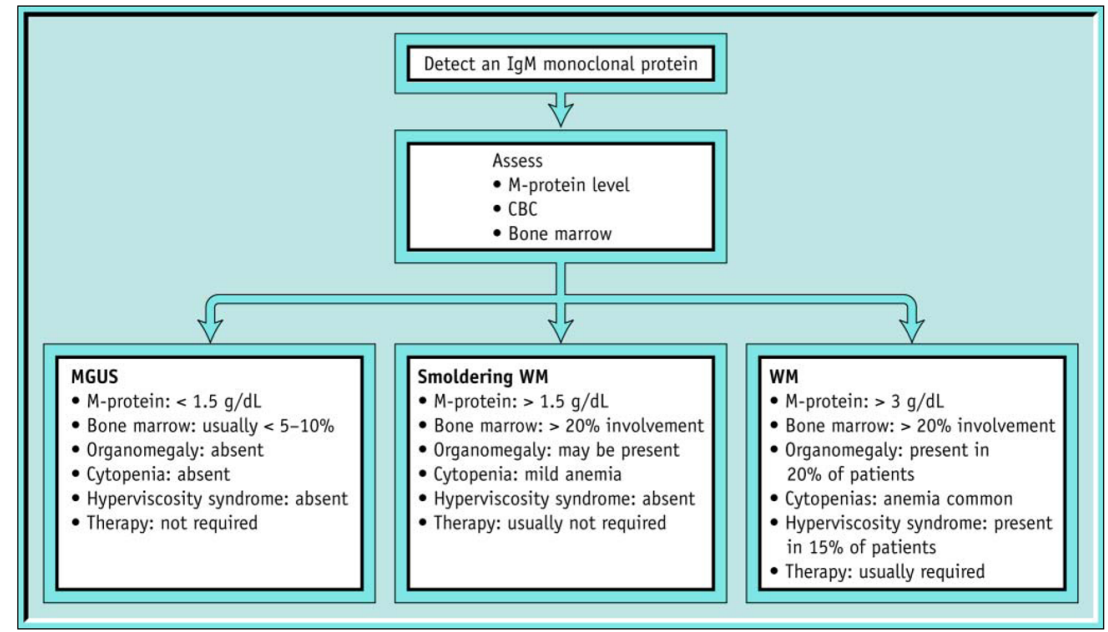

Figure 3.

Steps of diagnosis, differential diagnosis, prognosis, and treatment in patients with WM. CBC — complete blood count; Ig_immunoglobulin; MGUS — monoclonal gammopathy of undetermined significance; WM-Waldenström macroglobulinemia. 
Table 1

Therapeutic options in Waldenström macroglobulinemia

\begin{tabular}{|ll|}
\hline Therapeutic options & Types of agents \\
\hline Alkylator-based therapy & Single agent, such as chlorambucil, or combinations \\
Purine nucleoside analogs & Fludarabine or cladribine \\
Monoclonal antibodies & Rituximab \\
Others & Steroids and interferon \\
Investigational therapy & Stem cell transplantation (autologous and allogeneic) and thalidomide \\
Treatment of complications & Plasma exchange \\
Surgical interventions & Splenectomy rarely needed \\
Supportive care & Erythropoietin and red blood cell transfusions \\
\hline
\end{tabular}

